\title{
Rôle du calcium, des liaisons esters des diglycérides et d'un peptide de synthèse dans l'activation de la protéine kinase $\mathrm{C}$
}

\author{
C. Da Silva, M. Pilloud, I. Martelly et M. Castagna
}

Groupe de laboratoires de I'IRSC, 7 rue Guy-Mocquet, 94802 Villejuif Cedex, France

(14 Réunion du groupe Développement INRA, Clermont-Ferrand, 25-27 mai 1988)

\begin{abstract}
Résumé - La protéine kinase $C$, qui joue un rôle clé dans la voie de signalisation impliquant les polyphosphoinositides est activée par de nombreuses classes de ligands extracellulaires, notamment des neuromédiateurs, des hormones et des facteurs de croissance. Les diglycérides sont les médiateurs intracellulaires de l'activation de la protéine kinase $C$. Les promoteurs de tumeurs du type des esters de phorbol se substituent aux diglycérides sur leur site de liaison à la PK C et ainsi miment leurs effets. Les diglycérides actifs ont une configuration 1,2 sn et sont estérifiés par des acides gras saturés à courtes chaînes ou insaturés à longues chaînes. Les analogues alkylés des diglycérides avec une liaison éther en 1 sont inactifs, contrairement à ceux qui alkylés en 2 ont conservé une liaison ester en 1. L'activation de la protéine kinase $\mathrm{C}$ et la liaison du ${ }^{3} \mathrm{H}$-TPA à l'enzyme s'observent en présence de 0,5 mM EGTA. De plus, l'activation totale de l'enzyme est obtenue dans les plaquettes chargées avec un excès de Quin 2, avant leur stimulation par les esters de phorbol. Un peptide du site catalytique correspondant à la séquence $499-513$ a été synthétisé. II augmente l'affinité de la protéine kinase $C$ pour les histones. Ces résultats suggèrent que cette séquence de l'enzyme appartient au récepteur d'un autre peptide (résidus 19-36) qui lui joue le rôle d'un pseudosubstrat.
\end{abstract}

protéine kinase $\mathrm{C}-$ diglycérides $-\mathrm{Ca}^{2+}-$ site du pseudo-substrat

Summary - Role of calcium, diglyceride esters and a synthetic peptide in protein kinase C activation. Protein kinase $C$, which plays a significant role in the polyphosphoinositide pathway of transmembrane signaling, is activated by a large class of extracellular ligands including neurotransmitters, hormones and growth factors. Diacylglycerols are the intracellular mediators of protein kinase $C$ activation. Tumor promoting phorbol esters mimic the diacylglycerol action in binding to the same site. Active diacylglycerols have the 1,2 sn configuration and saturated short chain or unsaturated long chain fatty acids. Alkyl analogs of diacylglycerols were devoid of activity when an ether bond was present in position 1, whereas activity of the alkyl analog in position 2 was retained. Protein kinase $C$ activation and ${ }^{3} \mathrm{H}$-TPA binding to the enzyme occurred in the presence of $0.5 \mathrm{mM}$ EGTA. Moreover it has been shown in vivo that full activation of the enzyme was obtained in the intact platelets loaded with an excess of Quin 2, prior to stimulation by phorbol esters. A peptide (residues 499-513) was synthesized which enhanced the affinity of protein kinase $C$ for histone. It is suggested that it may be the receptor site for another peptide of the enzyme (residues 19 to 36) which behaves as a pseudosubstrate.

protein kinase $\mathrm{C}$ - diacylglycerols - $\mathrm{Ca}^{2+}-$ site of pseudosubstrate 


\section{INTRODUCTION}

Les mécanismes par lesquels les cellules captent, transfèrent et répondent aux signaux de l'environnement constituent une problématique majeure de la biologie. C'est la découverte de I'AMP cyclique et de sa cible moléculaire, la protéine kinase dépendante de l'AMP cyclique, qui a ouvert ce champ de recherche en décrivant les premiers éléments de la voie qu'empruntent certains neuromédiateurs et hormones, pour transférer leurs informations à l'intérieur de la cellule. Ces recherches se sont rapidement développées et particulièrement depuis la mise en évidence de la voie des polyphosphoinositides qu'empruntent de nombreux ligands extracellulaires, neuromédiateurs, hormones et facteurs de croissance. Cette voie, qui fait intervenir 2 seconds messagers à courte vie, l'inositol triphosphate $\left(\mathrm{IP}_{3}\right)$ et les diglycérides (DG), apparaît donc comme le site où s'exerce la régulation d'un grand nombre de fonctions cellulaires et de la prolifération. II est bien démontré que $\mathrm{IIP}_{3}$ mobilise le $\mathrm{Ca}^{2+}$ de ses sites de stockage, tandis que les DG activent la protéine kinase $C$. L'activation des processus régulés par $\mathrm{Ca}^{2+}$ et des phosphorylations catalysées par la protéine kinase, produisent la réponse au ligand extracellulaire (Berridge, 1984; Castagna, 1987; Nishizuka, 1986). Les promoteurs de tumeurs, tels que les esters de phorbol, activent également la protéine kinase $\mathrm{C}$ via leur liaison au site des DG.

Le propos de cette comminication est de contribuer à préciser la spécificité des $D G$ et le rôle de $\mathrm{Ca}^{2+}$ dans la réaction d'activation. De plus, nos recherches sur la fonction d'un peptide du site catalytique de la protéine kinase $C$ et son rôle possible dans la régulation de l'activation de l'enzyme seront présentées.

\section{MATÉRIELS ET MÉTHODES}

\section{Mesure in vitro de l'activité protéine ki- nase $C$}

L'enzyme a été préparée à partir de cerveau de souris comme décrit précédemment (Roghani et al., 1987). L'activité a été mesurée par une technique décrite par Couturier et al. (1984), modifiée comme suit : $0,4 \mu \mathrm{g}$ d'enzyme (activité $=30$ $\mu \mathrm{mol} 32 \mathrm{P}$ transférées $/ \mathrm{min} / \mathrm{mg}$ protéine) étant ajouté au milieu réactionnel et la réaction a été arrêtée par le transfert de $50 \mu \mathrm{L}$ sur des carrés de papier Whatman P81, et suivie de plusieurs lavages dans l'acide phosphorique à $1 \%$.

\section{Mesure de l'activité protéine kinase $C$ dans les plaquettes intactes}

Les plaquettes de lapin lavées selon la méthode de Baenziger \& Majerus (1974) ont été marquées par ${ }^{32 \mathrm{P}}$ avant traitement par les activateurs de la protéine kinase $C$ à tester. Les lipides neutres sont additionnés après sonication. Les phosphoprotéines sont analysées par électrophorèse (SDS-PAGE) suivie d'une autoradiographie.

\section{Synthèse des analogues de diglyce- rides}

La synthèse d'analogues alkylés du 1,2-racdidécanoylglycérol a été réalisée par Heymans et al. selon une technique déjà décrite (1987).

\section{Mesure de la liaison du $\left.{ }^{3} \mathrm{H}\right)$ TPA à la protéine kinase $C$}

La liaison du TPA à l'enzyme est mesurée selon la technique décrite par Ashendel et al. (1983). 
La réaction est stoppée par filtration des échantillons sur des filtres Whatman GF/C qui sont lavés 4 fois dans l'acétone froid avant la mesure de radioactivité.

\section{Traitement des plaquettes avec le Quin 2}

Les plaquettes sont incubées en présence de $0,1 \mathrm{mM}$ Quin 2, un chélateur du $\mathrm{Ca}^{2+}$. Pour prévenir l'influx de $\mathrm{Ca}^{2+}$ provoqué par la diminution de $\mathrm{Ca}^{2+}$ intracellulaire, 0,2 $\mathrm{mM}$ d'EGTA sont ajoutés au milieu d'incubation. Après 20 et 40 min, les cellules sont stimulées par $0,1 \mu \mathrm{g} / \mathrm{mL}$ de TPA pendant 2 min en présence de ${ }^{32} \mathrm{P}$. Des plaquettes traitées seulement avec le DMSO, le solvant du Quin 2, servent de témoins.

Les protéines des 2 lots de plaquettes sont soumises à une électrophorèse en gel de polyacrylamide-SDS. Une autoradiographie est ensuite effectuée.

\section{RÉSULTATS}

\section{Spécificité des diglycérides}

Nous avons testé l'effet sur l'activation de la protéine kinase $\mathrm{C}$ des analogues suivants :

- 1-O-décyl,2-O-décanoyl-rac-glycérol(I)

- 1-O-décanoyl,2-O-décyl-rac-glycérol (II)

- 1-0,2-O-didécyl-rac-glycérol (III)

-1-O-décyl,3-O-décanoyl-rac-glycérol

(IV)

- 1-O-hexadécyl,2-O-acétylglycérol (V)

- 1-O-octadécyl,2-O-acétylglycérol (VI).

La Figure 1 montre que la liaison éther en 1 entraîne la perte de l'activité du composé (l et III et IV). En revanche, cette liaj- son en 2 (II) n'inhibe pas l'interaction avec l'enzyme, quoiqu'elle augmente son $\mathrm{Ka}$ apparent. Les précurseurs du PAF-acéther ( $V$ et $V I$ ) se révèlent dépourvus d'activité (résultats non illustrés).

Pour compléter ces tests in vitro, l'action du composé II sur la réponse plaquettaire a été examinée. Après activation, la protéine kinase $\mathrm{C}$ phosphoryle dans ces cellules un certain nombre de protéines, dont une de $43 \mathrm{kDa}$, représente son substrat majeur. Cette protéine est utilisée comme un index de l'activité de l'enzyme dans ces cellules. L'autoradiogramme des protéines de plaquettes isolées par SDS-PAGE montre que l'analogue alkylé en 2 active nettement la protéine kinase $\mathrm{C}$ in vivo, quoique moins intensément que le diester correspondant (Fig. 2). II peut aussi être

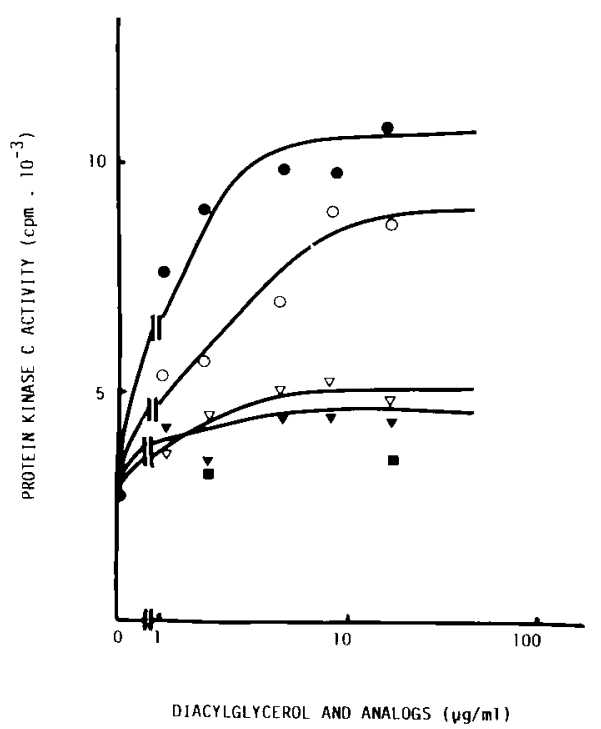

Fig. 1. Activation de la protéine kinase $C$ en réponse au 1,2-didécanoylglycérol (O) et à ses analogues alkylés : 1-O-décyl,2-O-décanoyl-glycérol (V); 1-0-décanoyl,2-0-décylglycérol (O); 1-2-0didecylglycérol $(\nabla) ; 1$ 1-O-décyl,3-O-décanoylglycérol $(\square)$. Le milieu réactionnel contenait 0,5 mM EGTA (d'après Heymans et al., 1987). 
observé que la demi-vie de l'état phosphorylé de la protéine $43 \mathrm{kDa}$ est proche de $30 \mathrm{~min}$ et n'est pas modifiée par le R59022, un inhibiteur de la diglycéride kinase.

\section{Rôle du Ca2+ dans l'activité de la pro- téine kinase $C$}

\section{Dépendance in vitro de l'activation de la protéine kinase $\mathrm{C}$ à l'égard du $\mathrm{Ca}^{2+}$}

La courbe dose-réponse de l'activation de la protéine kinase $C$ par la phosphatidylsérine en présence de $2,5 \mathrm{mM}$ de $\mathrm{CaCl}_{2}$ et de $0,5 \mathrm{mM}$ EGTA est montrée sur la Fig. $3 A$. II apparaît clairement que la réaction peut se produire en présence d'EGTA, mais avec une efficacité légèrement réduite. En parallèle, les capacités de liaison des esters de phorbol ont été comparées. Là encore, I'EGTA permet la réaction, mais il semble cependant qu'aux faibles concentrations de phosphatidylsérine $\mathrm{Ca}^{2+}$ stabilise la liaison (Fig. 3B).

\section{Dépendance à l'égard du $\mathrm{Ca}^{2}+$ in vivo}

Afin de rechercher si le $\mathrm{Ca}^{2+}$ est nécessaire à l'activation de l'enzyme in vivo, nous avons chargé les plaquettes de lapin avec un excès de Quin 2, un complexant du $\mathrm{Ca}^{2+}$. L'autoradiogramme des cellules traitées au TPA et témoins ne révèle aucune différence dans l'intensité de marquage de la protéine $43 \mathrm{kDa}$ (Fig. 4). No-

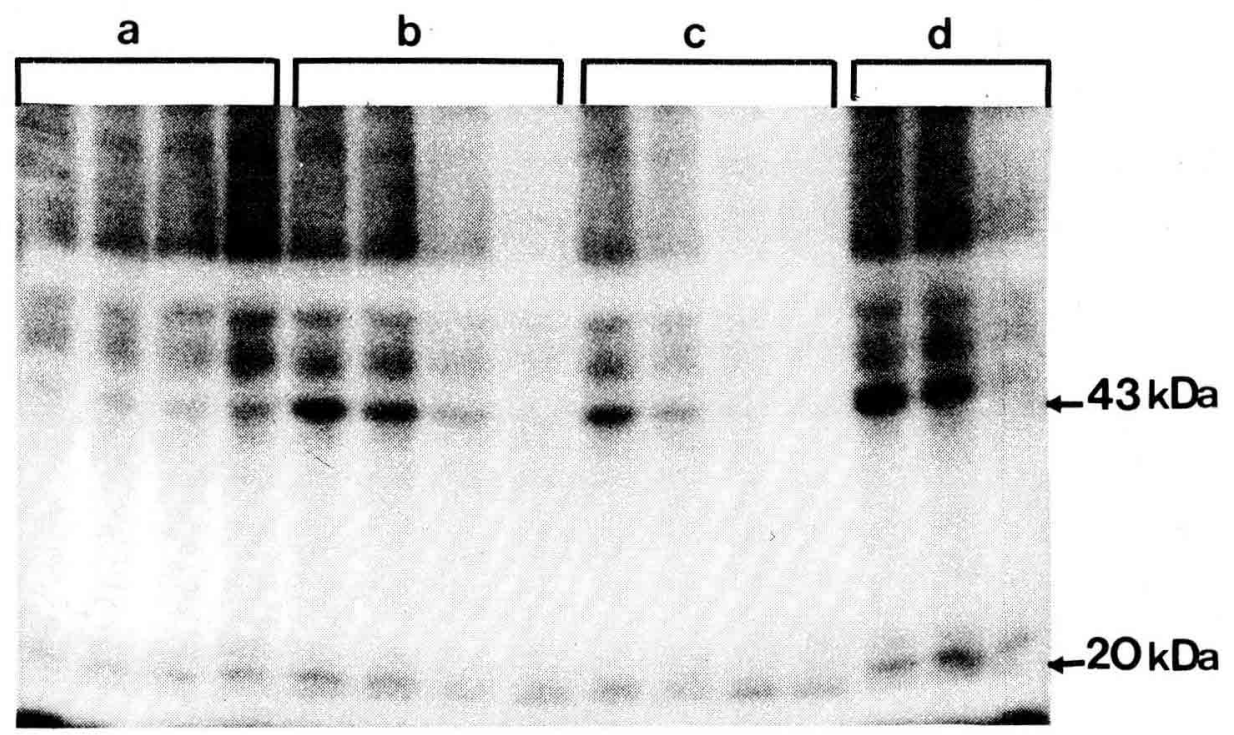

Fig. 2. Autoradiogramme des protéines de plaquettes prémarquées au ${ }^{32} \mathrm{P}$. Les cellules étaient incubées à $37{ }^{\circ} \mathrm{C}$ dans la solution Tyrode (puits a) contenant soit $100 \mu \mathrm{g} / \mathrm{mL}$ de 1-0-décanoyl-2-Odécylglycérol (puits b), soit $100 \mu \mathrm{g} / \mathrm{mL}$ de 1-O-décanoyl-2-O-décylglycérol + 0,1 mM R59022 (puits c) soit $100 \mu \mathrm{g} / \mathrm{mL}$ de 1-2-O-didécanoylglycérol (puits d) pendant $1 \mathrm{~min}, 15 \mathrm{~min}, 30 \mathrm{~min}$ et $1 \mathrm{~h}$ (puits de gauche à droite) à l'exception des puits d dont les cellules étaient incubées pendant 1, 15 et 30 min (d'après Heymans et al., 1987). 

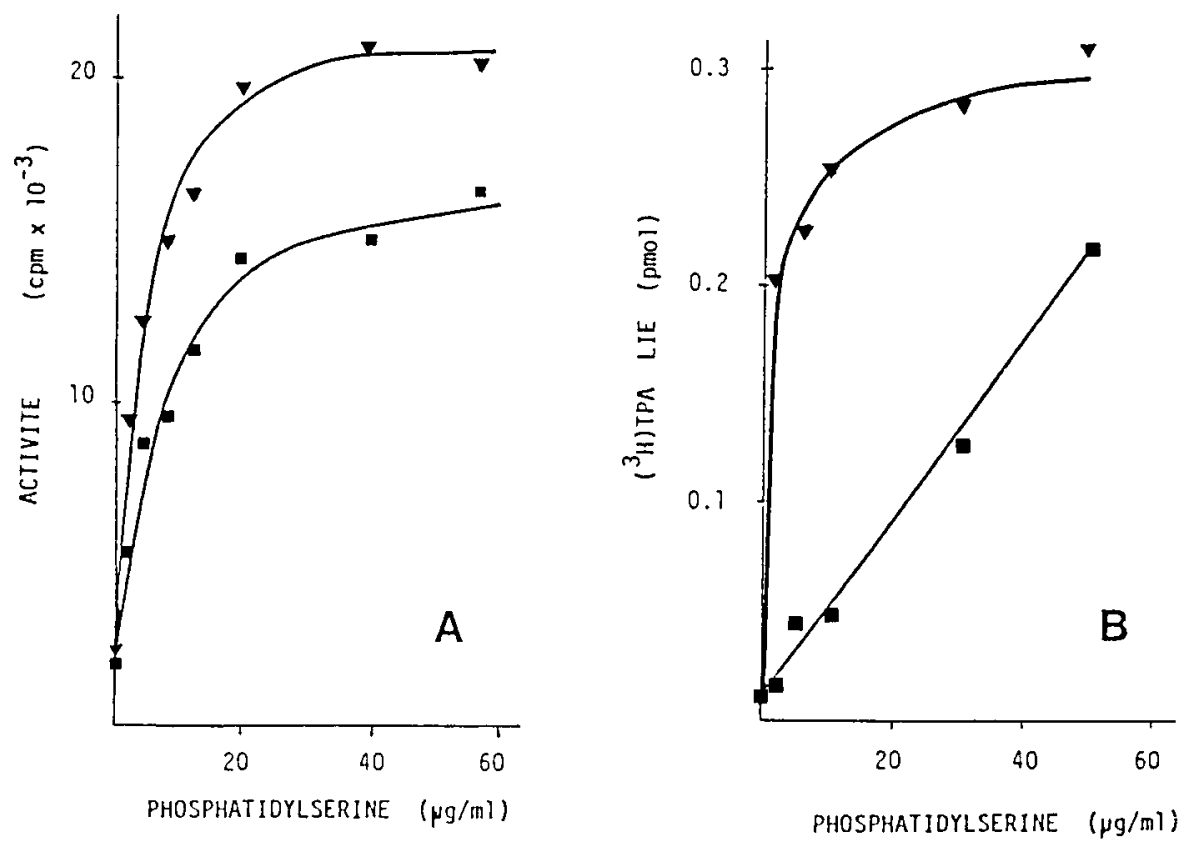

Fig. 3. Activation de la protéine kinase $\mathrm{C}$ et liaison du ${ }^{3} \mathrm{H}$-TPA en présence $(\nabla)$ ou en l'absence $(\boldsymbol{\nabla})$ de $2,5 \mathrm{mM}$ de $\mathrm{CaCl}_{2}$ en réponse à des concentrations croissantes de phosphatidylsérine. Fig. $3 \mathrm{~A}$ : activité de l'enzyme mesurée par la phosphorylation des histones; Fig. 3B : liaison spécifique du ${ }^{3} \mathrm{H}$-TPA à l'enzyme.

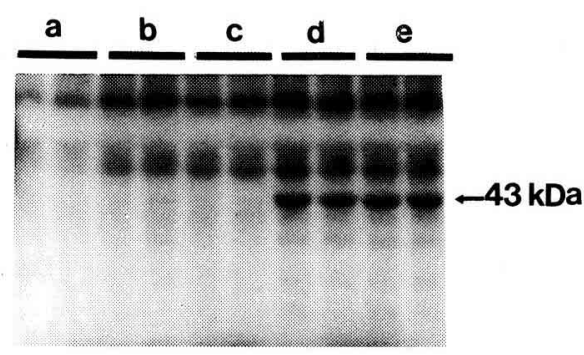

Fig. 4. Autoradiogramme des protéines de plaquettes prémarquées au ${ }^{32} \mathrm{P}$. Les cellules $(2 \times$ $10^{8}$ par essai) étaient incubées à $37^{\circ} \mathrm{C}$ pendant 20 min (puits de gauche) ou $40 \mathrm{~min}$ (puits de droite) dans une solution Tyrode contenant 0,2 $\mathrm{mM}$ EGTA (puits a), ainsi que 0,1 mM Quin 2 (puits b) ou $10 \%$ DMSO (puits c), ou $0,1 \mathrm{mM}$ Quin $2+0,1 \mu \mathrm{g} / \mathrm{ml}$ TPA (puits d) ou $10 \%$ DMSO $+0,1 \mu \mathrm{g} / \mathrm{ml}$ TPA (puits e). Dans les échantillons d et et, le TPA était ajouté dans les 2 dernières min de l'incubation. tons qu'en conditions expérimentales, la quantité de $\mathrm{Ca}^{2}+$ libre peut être évaluée à $20 \mathrm{nM}$ dans les plaquettes en présence de $0,1 \mathrm{mM}$ de Quin 2.

\section{Fonction du peptide correspondant à la séquence comprise entre les résidus 499 et 513 (isoforme $\beta$ )}

Le peptide (PA 87) est composé de 15 acides aminés dont la séquence se situe dans le site catalytique de l'enzyme. La présence du peptide à des concentrations croissantes dans le milieu réactionnel augmente la phosphorylation des histones 
(Fig. 5). La réaction est favorisée par le $\mathrm{Ca}^{2+}$. Le peptide n'affecte pas l'autophosphorylation de l'enzyme (résultat non illustré). Le peptide augmente l'affinité de la protéine kinase $\mathrm{C}$ pour le substrat. Nous avons trouvé que le $\mathrm{Km}$, dont la valeur est de 6,3 $\mu \mathrm{M}$ en l'absence du peptide, est diminué par un facteur d'environ 3 quand le peptide est présent à la concentration de $120 \mu \mathrm{g} / \mathrm{mL}$.

\section{DISCUSSION ET CONCLUSIONS}

Nous avons montré que les diglycérides actifs sur l'activité de la protéine kinase $C$ ont une configuration 1,2 sn (Couturier et al., 1984) et sont estérifiés par des acides gras insaturés à longue chaîne ou saturés à courte chaîne. Les stéréo-isomères 2,3 sn sont inactifs. Les résultats présentés ici

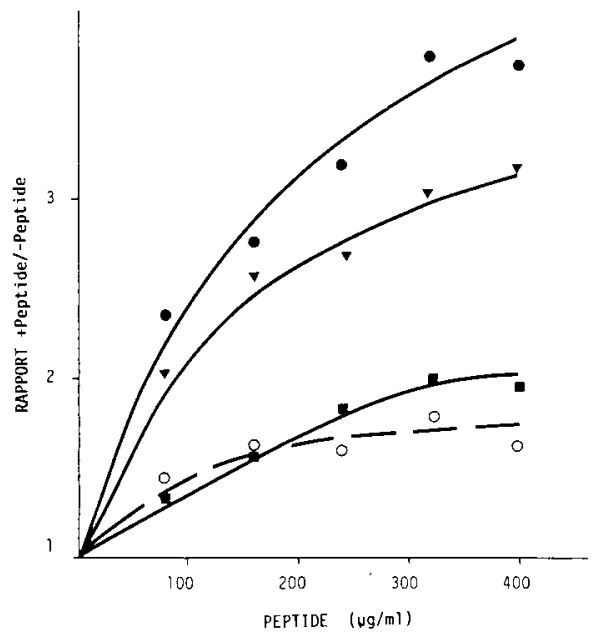

Fig. 5. Activation de la protéine kinase $C$ par des doses croissantes de peptide à des concentrations variables d'histones. Le milieu réactionnel contenait $0,5 \mathrm{mM}$ de $\mathrm{CaCl}_{2}$ et des histones à la concentration de $40 \mu \mathrm{g} / \mathrm{mL}(\boldsymbol{D}), 120 \mu \mathrm{g} / \mathrm{mL}(\nabla)$, $200 \mu \mathrm{g} / \mathrm{mL}$ (@) ou $0,5 \mathrm{mM}$ d'EGTA et $200 \mu \mathrm{g} / \mathrm{mL}$ d'histones (O). établissent que la liaison ester en position 1 est indispensable à l'activité des diglycérides.

La protéine kinase $C$ n'apparaît pas dépendante de $\mathrm{Ca}^{2+}$ pour son activité : en présence d'EGTA, l'enzyme peut fixer le TPA et s'activer. De même, les expériences réalisées dans les plaquettes in vivo stimulées par l'ester de phorbol TPA, dans des conditions où le taux en $\mathrm{Ca}^{2+}$ libre était considérablement abaissé, ne semblent pas vérifier la dépendance de l'enzyme à l'égard de $\mathrm{Ca}^{2+}$.

Le peptide PA 87 se comporte comme un activateur de la protéine kinase $C$. Récemment, House \& Kemp (1987) ont mis en évidence l'existence d'une séquence de la région régulatrice de l'enzyme qui inhibe son activité. II a suggéré que cette séquence bloque le site catalytique par repliement de la molécule. Nous explorons actuellement la possibilité que le peptide PA 87 soit le récepteur de la séquence inhibitrice.

\section{RÉFÉRENCES}

Ashendel C.L., Staller J.M. \& Boutwell R.K. (1983) Identification of a calcium and phospholipid-dependent phorbol ester binding activity in the soluble fraction of mouse tissues. Biochem. Biophys. Res. Commun. 111, 341-345

Baenzinger H.L. \& Majerus P.W. (1974) Isolation of human platelets and platelet surface membranes. Methods Enzymol. 31, 149-155

Berridge M.J. (1984) Inositol triphosphate and diacylglycerol as second messengers. Biochem. J. $220,345-360$

Castagna M. (1987) Phorbol esters as signal transducers and tumor promoters. Biol. Cell. 59, 3-14

Couturier A., Bazgar S. \& Castagna M. (1984) Further characterization of tumor promotermediated activation of protein kinase C. Biochim. Biophys. Res. Commun. 121, 448-455 
Heymans F., Da Silva C., Marrec N., Godfroid J.J. \& Castagna M. (1987) Alkayl analogs of diacylglycerol as activators of protein kinase $C$. Fed. Eur. Biochem. Soc. Lett. 218, 35-40 House C. \& Kemp V.B.E. (1987) Protein kinase C contains a pseudo substrate protope in its regulatory domain. Science 238, 1726-1728
Nishizuka Y. (1986) Studies and perspectives of protein kinase C. Science 233, 305-312

Roghani M., Da Silva C. \& Castagna M. (1987) Tumor promotor chloroform is a potent protein kinase $\mathrm{C}$ activator. Biochim. Biophys. Res. Commun. 142, 738-742 